\title{
Virtuelle Gruppendynamik
}

\author{
Monique Janneck
}

In dem Maße, in dem computergestützte Kommunikation und Kooperation den Alltag durchdringen - sei es bei der Arbeit, beim Lernen oder im persönlichen Bereich - werden soziale Prozesse virtualisiert und über Kommunikationsmedien vermittelt. Die Beiträge in diesem Heft befassen sich mit verschiedensten Aspekten solch virtueller Gruppendynamik.

Der erste Beitrag von Julia Gallenkamp, Arnold Picot, Isabell Welpe und Marcus Drescher (Die Dynamik von Führung, Vertrauen und Konflikt in virtuellen Teams) gibt einen Überblick über den Forschungsstand zu virtuellen Teams, speziell im Hinblick auf Führung, Vertrauen und Konflikte bei virtueller Zusammenarbeit. Die Autoren systematisieren zunächst Merkmale und Ausprägungen virtueller Teams und stellen methodische Herausforderungen bei der Erforschung virtueller Teams dar. Anschließend stellen die Autoren summarisch Ergebnisse eigener Studien aus einem größeren Forschungsprojekt über virtuelle Teams vor, eingebettet in eine Darstellung des Forschungsstandes. Dabei widmen sich die Autoren insbesondere Konflikten, Vertrauen und Führung in virtuellen Teams. Zudem werden kulturelle Einflüsse thematisiert.

„Auch im Netzwerk tobt das Leben“ betitelt Henning Staar seinen Beitrag, in dem er sich mit der Relevanz mikropolitischer Prozesse in virtuellen Kooperationsverbünden beschäftigt. Der Beitrag ist konzeptioneller Natur und hat zum Ziel, Mikropolitik als einflussreiches organisationstheoretisches Konzept auf inter-organisationale, speziell virtuelle Kooperationsverbünde zu übertragen: Vor dem Hintergrund einer stetigen Zunahme von Netzwerkkooperationen - insbesondere von Freiberuflern und kleinen und mittleren Unternehmen - ist die Frage der Steuerung solcher Verbünde und ein Verständnis ihrer inneren Dynamik und Erfolgskriterien von hohem wissenschaftlichem und praktischem Interesse. Der Beitrag gibt eine grundlegende Einführung in das Thema Mikropolitik und stellt den Stand der Forschung speziell im Hinblick auf Netzwerke dar. Auf dieser Grundlage entwickelt der Autor ein Modell mikropolitischer Prozesse in virtuellen Netzwerken, das Bedingungen mikropolitischen Handelns, mikropolitische Verhaltensweisen, per-

Online publiziert: 04.11 .2010

(C) VS Verlag für Sozialwissenschaften 2010

Dr. rer. nat. M. Janneck $(\bowtie)$

Fakultät 4, Arbeits- und Organisationspsychologie,

Universität Hamburg, Von-Melle-Park 11,

20146 Hamburg, Deutschland

E-Mail: monique.janneck@uni-hamburg.de 
sonenbezogene Charakteristika sowie Folgen mikropolitischen Handelns integriert und stellt erste eigene empirische Forschungsergebnisse vor.

Der nachfolgende Beitrag von Isabella von Wissmann (Jenseits der Regelbarkeit? Nutzung informeller Dynamiken von Kommunikation und Gruppenrollen zur Steuerung virtueller Gruppen) thematisiert Kommunikations- und Gruppenstrukturen in virtuellen Teams. Die Autorin nimmt dabei in einer facettenreichen empirischen Untersuchung insbesondere die formellen und informellen Rollen, die von den Teammitgliedern ausgefüllt werden, in den Blick. Aus den Ergebnissen leitet sie Empfehlungen zur Steuerung virtueller Teams sowie für die Gestaltung von Veränderungsprozessen im Kontext virtueller Teams, wie beispielsweise die Einführung neuer Informations- und Kommunikationstechnologien, ab.

Nina Haferkamp und Nicole Krämer (Krisenkommunikation in virtuellen Realitäten - Evaluation eines Serious Games zum Training von Soft Skills in Krisenstäben) befassen sich in ihrem Artikel mit der Nutzung von Computerspielen zum Training von Gruppenund Kommunikationssituationen und stellen ein virtuelles Trainingsspiel zur Simulation der Arbeit von Krisenstäben in Katastrophensituationen vor, dessen Ziel es ist, effiziente Kommunikationsabläufe und Entscheidungsprozesse in Notfallsituationen einzuüben und dabei das Training kostengünstiger und organisatorisch weniger aufwändig zu gestalten als bei herkömmlichen Schulungen und Simulationen. Zur Entwicklung des Spiels wurden Erkenntnisse zu gruppendynamischen Phänomenen wie Gruppendenken und Soziales Faulenzen herangezogen. Die Ergebnisse zweier Studien zur Evaluation des Trainingsspiels werden vorgestellt, die erwarten lassen, dass das Spiel erfolgreich zur Simulation und zum Training von komplexen Kommunikations- und Entscheidungssituationen in Gruppen genutzt werden kann.

Der abschließende Beitrag von Philipp Taesler und Monique Janneck (Emoticons und Personenwahrnehmung: Der Einfluss von Emoticons auf die Einschätzung unbekannter Kommunikationspartner in der Online-Kommunikation) befasst sich mit Wirkungen textbasierter Online-Kommunikation, die trotz vielfältiger technischer Möglichkeiten nach wie vor einen Großteil der computervermittelten Kommunikation ausmacht. In einem Online-Experiment wurde gezeigt, dass sich die weit verbreitete Verwendung grafischer Darstellungen zum Ausdruck von Gefühlslagen und nonverbalen Kommunikationsaspekten - sogenannte Emoticons - auf die Einschätzung des Online-Kommunikationspartners auswirkt. Die Thematik ist sowohl aus Forschungs- als auch aus Praxissicht relevant, um beispielsweise als passend bzw. unpassend empfundene Kommunikationsstile in verschiedenen Kontexten zu identifizieren und stellt somit eine wichtige Grundlage für die Gruppenkommunikation dar.

Diese fünf Beiträge, die das Thema „Virtuelle Gruppendynamik“ aus unterschiedlichen Blickwinkeln beleuchten, zeigen damit auf, wie facettenreich dieses Forschungs- und Praxisfeld ist. Interdisziplinäre Ansätze, die etwa die Gestaltung der Kommunikationsmedien vor dem Hintergrund psychologischer Theorien und Befunde zum Verhalten in virtuellen Gruppen thematisieren, scheinen vor diesem Hintergrund besonders vielversprechend für die weitere Forschung. 